\title{
Downregulation of CD147 expression by RNA interference inhibits HT29 cell proliferation, invasion and tumorigenicity in vitro and in vivo
}

\author{
RUI LI ${ }^{1,2^{*}}$, YUQIN PAN ${ }^{2 *}$, BANGSHUN HE ${ }^{2}$, YEQIONG XU², TIANYI GAO ${ }^{2}$, \\ GUOQI SONG ${ }^{2}$, HUILING SUN ${ }^{1,2}$, QIWEN DENG ${ }^{2}$ and SHUKUI WANG ${ }^{2}$ \\ ${ }^{1}$ Department of Life Sciences, Nanjing Normal University, Nanjing, Jiangsu $210006 ;{ }^{2}$ The Central Laboratory \\ of Nanjing First Hospital, Nanjing Medical University, Nanjing, Jiangsu 210006, P.R. China
}

Received July 18, 2013; Accepted September 2, 2013

DOI: 10.3892/ijo.2013.2108

\begin{abstract}
We investigated the effect of CD147 silencing on HT29 cell proliferation and invasion. We constructed a novel short hairpin RNA (shRNA) expression vector pYr-mir30-shRNA. The plasmid was transferred to HT29 cells. The expression of CD147, MCT1 (lactate transporters monocarboxylate transporter 1) and MCT4 (lactate transporters monocarboxylate transporter 4) were monitored by quantitative PCR and western blotting, respectively. The MMP-2 (matrix metalloproteinase-2) and MMP-9 (matrix metalloproteinase-9) activities were determined by gelatin zymography assay, while the intracellular lactate concentration was determined by the lactic acid assay kit. WST- 8 assay was used to determine the HT29 cell proliferation and the chemosensitivity. Invasion assay was used to determine the invasion of HT29 cells. In addition, we established a colorectal cancer model, and detected CD147 expression in vivo. The results showed that the expression of CD147 and MCT1 was significantly reduced at both mRNA and protein levels, and also the activity of MMP-2 and MMP-9 was reduced. The proliferation and invasion were decreased, but chemosensitivity to cisplatin was increased. In vivo, the CD147 expression was also significantly decreased, and reduced the tumor growth after CD147 gene silencing. The results demonstrated that silencing of CD147 expression inhibited the proliferation and invasion, suggesting CD147 silencing might be an adjuvant gene therapy strategy to chemotherapy.
\end{abstract}

Correspondence to: Dr Shukui Wang, Nanjing First Hospital, Nanjing Medical University, Nanjing, Jiangsu 210006, P.R. China E-mail: shukwang@163.com

${ }^{*}$ Contributed equally

Key words: RNA interference, colorectal cancer, HT29 cells, CD147

\section{Introduction}

Colorectal cancer is the third most common cancer in men $(10.0 \%$ of the total, $\sim 663,000$ cases $)$ and the second in women $(9.4 \%$ of the total, $\sim 570,000$ cases) worldwide (1). Approximately 608,000 colorectal cancer deaths were estimated worldwide, accounting for $8 \%$ of all cancer deaths, making it the fourth most common cause of death from cancer. The traditional treatment of colorectal cancer is generally drugs, radiotherapy and chemotherapy, but the effect of these methods are not satisfactory, the mortality rate of colorectal cancer still remains high. The study of Center et al (2) indicated that the mortality of colorectal cancer had also been increasing because of tumor relapse and metastasis. However, carcinogenesis is a complicated biological process, and the molecular mechanisms, metastasis phenotype, pathways and regulating genes are not well known (3). Therefore, better understanding of molecular mechanisms underlying proliferation, invasion and survival of colorectal cancer are critical for the development of optimal therapeutic modalities.

CD147 (also called EMMPRIN, basigin, tumor cell derived collagenase stimulatory factor, or human leukocyte activation-associated M6 antigen), is a 43-66-kDa multifunctional glycosylated transmembrane protein, which belongs to the immunoglobulin superfamily (4-7). The protein of CD147 is highly expressed on the cell surface of many tumor cells such as, oral, breast, lung, bladder, kidney, laryngeal, pancreatic, gastric, colorectal cancer, glioma, lymphoma and melanoma (8-13), and was correlated with tumor progression and invasion $(14,15)$ and could also stimulate tumor cells to produce matrix metalloproteinases (MMPs), a family of zinc-dependent endopeptidases including $>25$ members, specifically MMP-2 and MMP-9 $(5,16)$. The MMPs are one of the important factors of tumor invasion and metastasis (17). Previous studies showed that CD147 could promote the generation of a MMP complex by endothelial cells, which modified the tumor cell pericellular matrix concentrating at tumor cell surface to promote tumor cell invasion $(18,19)$. In addition, CD147 was also able to influence lactate transport and glycolysis by its association with lactate transporters 
monocarboxylat transporter (MCT), specifically MCT-1 and MCT-4 (20). MCT-1 and MCT-4 are two members of the proton-linked monocarboxylate (lactate) transporter family, playing a fundamental role in metabolism. CD147 is essential in transporting MCT1 and MCT4 to plasma membrane (21). CD147 was also involved in multidrug resistance of cancer cells via hyaluronan-mediated activation of ErbB2 signaling and survival pathway activity, and multidrug resistance and tumor invasiveness might be linked during the progression of malignant disease $(22,23)$, but the function and mechanism of CD147 remain elusive on proliferation, invasion, metastasis and multidrug resistance of colorectal cancer.

The studies of Zhu et al (12) showed that CD147 expression was high in colorectal cancer and and was associated with the colorectal development, and with poor prognosis. The molecular mechanisms involved and the role of CD147 in colorectal cancer, however, remained poorly understood. To determine the role of CD147 in invasiveness, metastasis, growth and survival of colorectal cancer, we used RNA interference (RNAi) technique to knock down the expression of CD147 in HT29 cells, and investigated its roles on proliferation, invasion and the chemosensitivity of colorectal cancer cells.

\section{Materials and methods}

Cell culture. HT29 cells, a human colorectal cancer cell line, was provided from the Shanghai Cell Collection, the Chinese Academy of Sciences. Cells were maintained in DMEM (Gibco BRL, Grand Island, NY, USA) with $10 \%$ fetal bovine serum (FBS), $100 \mathrm{U} / \mathrm{ml}$ of penicillin and $100 \mathrm{~g} / \mathrm{ml}$ of streptomycin (Gibco BRL) in a $5 \% \mathrm{CO}_{2}$ humidified atmosphere at $37^{\circ} \mathrm{C}$.

Design of pYr-mir30-shRNA plasmid construction. The vector pYr-mir30-shRNA was used to generate short hairpin RNA (shRNA) specific for CD147 by selecting the 808-828 fragment as the RNAi target site, and the scrambled control sequence was also synthesized as shown in Table I. These oligonucleotides were annealed and subcloned into the BsaI sites of the vector. These recombinant vectors were designated as pYr-mir30-shRNA-control and pYr-mir30-shRNA, respectively. The vector of pYr-mir30-shRNA included the EGFP gene sequence, so the EGFP protein expression can reflect the CD147 protein expression. All the cloned genes were confirmed by DNA sequencing.

Transient and transfection screening. HT29 cells were plated in 6-well plates at a density of $3 \times 10^{5}$ cells per well and incubated in $2 \mathrm{ml}$ of growth medium without antibiotics. When the cells reached $80 \%$ confluence after $24-\mathrm{h}$ incubation, cells were transferred with pYr-mir30-CD147shRNA-control and pYr-mir30-CD147-shRNA, respectively, using Lipofectamine 2000 (Invitrogen-Life Technologies, Carlsbad, CA, USA) according to the manufacturer's instructions. Forty-eight hours after transfection, HT29 cells were diluted 1:10 for passage and neomycin resistance clones were selected in the medium containing $600 \mu \mathrm{g} / \mathrm{ml} \mathrm{G} 418$ (Gibco-BRL) for 2 weeks. The positive clones were picked and expanded to establish cell lines in $300 \mu \mathrm{g} / \mathrm{ml} \mathrm{G} 418$. The stable transfection cell clones, designated as HT29/shRNA-control,
HT29/shRNA, were verified by quantitative real-time RT-PCR and western blot analysis.

Quantitative real-time PCR assay. Total cellular RNA was extracted using TRIzol reagent (Invitrogen), according to the manufacturer's instructions and reverse transcribed into cDNA using PrimeScript RT reagent kit Perfect Real (Takara). First the cDNA was quantified in a 1:10 dilution on a spectrophotometer. CD147 mRNA expression was evaluated by RT-PCR on an ABI PRISM 7500 real-time PCR apparatus (Applied Biosystems, USA) with SYBR Premix Ex $\mathrm{Taq}^{\mathrm{TM}}$ II. The primer sequences used for CD147, MCT1, MCT4 and $\beta$-actin are listed in Table II. The conditions for real-time PCR were: $95^{\circ} \mathrm{C}$ for $30 \mathrm{sec}$, then 40 cycles at $95^{\circ} \mathrm{C}$ for $5 \mathrm{sec}$, and $60^{\circ} \mathrm{C}$ for $34 \mathrm{sec}$. The mRNA level for CD147 of each sample was normalized to $\mathrm{Ct}$ values of the $\beta$-actin amplified from the same sample, $\Delta \mathrm{Ct}=\mathrm{Ct}_{\mathrm{CD} 147}-\mathrm{Ct}_{\beta \text {-actin }}$ and the $2^{-\Delta \Delta \mathrm{Ct}}$ method was used to calculate gene expression change. Samples were measured in triplicates to ensure the reproducibility of the results.

Western blot analysis. Western blot analysis was performed to evaluate CD147, MCT1 and MCT4 protein levels. The cultured tumor cells were washed three times with ice-cold PBS, then the cells were suspended in lysis buffer $[150 \mathrm{mM}$ $\mathrm{NaCl}, 50 \mathrm{mM}$ Tris-HCl (pH 7.4), $1 \mathrm{mM} \mathrm{MgCl}{ }_{2}, 100 \mu \mathrm{g} / \mathrm{ml}$ PMSF, $1.0 \%$ Triton X-100] on ice for $30 \mathrm{~min}$. Cell lysates were then collected after centrifugation at $12,000 \mathrm{rpm}$ for $5 \mathrm{~min}$ at $4^{\circ} \mathrm{C}$. Equal amounts $(30 \mu \mathrm{g})$ of lysate proteins were separated on $10 \%$ SDS-PADE gels, and transferred to a polyvinylidene difluoride (PVDF) membrane. After blocking with 5\% non-fat dry milk in TBST buffer (10 mM Tris- $\mathrm{HCl}, \mathrm{pH} 7.5,150 \mathrm{mM}$ $\mathrm{NaCl}$, and $0.05 \%$ Tween-20) for $2 \mathrm{~h}$ at room temperature, the membrane was probed with mouse anti-CD147 primary antibodies (1:500), rabbit anti-MCT1 primary antibodies (1:500), rabbit anti-MCT4 primary antibodies (1:500) and rabbit anti-human $\beta$-actin primary antibodies (1:500) incubated at room temperature for $2 \mathrm{~h}$, followed by incubation in a 1:2,000 dilution of secondary antibodies conjugated to horseradish peroxidase (Santa Cruz Biotechnology, Santa Cruz, CA, USA) for $1 \mathrm{~h}$ at room temperature. Protein bands were detected using ECL detection system (Boster, Wuhan, China). Western blot experiments were performed at least three times.

Gelatin zymography assay. Cells were cultured in serum-free DMEM medium for $24 \mathrm{~h}$, and then harvested in conditioned medium. The gelatinolytic activity of MMP-2 and MMP-9 in the conditioned medium was assayed by electrophoresis on $10 \%$ polyacrylamide gels containing $1 \mathrm{mg} / \mathrm{ml}$ of gelatin. PAGE gels were run at $100 \mathrm{~V}$ in stacking gels, and $100 \mathrm{~mA}$ in separating gels, washed in $2.5 \%$ Triton X-100 twice every $40 \mathrm{~min}$, and then incubated for $16 \mathrm{~h}$ at $37^{\circ} \mathrm{C}$ in activation buffer (50 mM Tris- $\mathrm{HCl}$, pH 7.6, $5 \mathrm{mM} \mathrm{CaCl}_{2}, 0.02 \%$ Brij-35). After reaction, the gels were stained with Coomassie Brilliant Blue $\mathrm{R}-250$ for $3 \mathrm{~h}$ and destained for $30 \mathrm{~min}$ in $20 \%$ methanol and $10 \%$ acetic acid. White lysis zones indicating gelatin degradation were revealed. This experiment was repeated at least three times.

Intracellular lactate concentration assay. We used a lactic acid assay kit (KeyGen Biotech Co., Ltd., Nanjing, China) to 
Table I. Sequences of the designed CD147 specific shRNAs.

shRNAs

shRNA-control

shRNA-control

shRNA

shRNA
Sequence

5'-GATCCACTACCGTTGTTATAGGTGTTCAAGAGA

CACCTATAACAACGGTAGTTTTTTTGGAAA-3'

5'-AGCTTTTCCAAAAAAACTACCGTTGTTATAGGT

GTCTCTTGAACACCTATAACAACGGTAGTG-3'

5'-GATCCGTGACAAAGGCAAGAACGTCTTCAAGA

GAGACGTTCTTGCCTTTGTCATTTTTTGGAAA-3'

5'-AGCTTTTCCAAAAAATGACAAAGGCAAGAACG

TCTCTCTTGAAGACGTTCTTGCCTTTGTCACG-3
Table II. Primers of CD147, MCT1, MCT4 and $\beta$-actin for real-time PCR.

\begin{tabular}{lll}
\hline Target & \multicolumn{1}{c}{ Primers } \\
\hline CD147 & Sense: & 5'-CCATGCTGGTCTGCAAGTCAG-3' \\
& Antisense: & 5'-CCGTTCATGAGGGCCTTGTC-3' \\
MCT1 & Sense: & 5'-CACTTAAAATGCCACCAGCA-3' \\
& Antisense: & 5'-AGAGAAGCCGATGGAAATGA-3' \\
MCT4 & Sense: & 5'-GTTGGGTTTGGCACTCAACT-3' \\
& Antisense: & 5'-GAAGACAGGGCTACCTGCTG-3' \\
3-actin & Sense: & 5'-CTGGAACGGTGAAGGTGACA-3' \\
& Antisense: & 5'-AAGGGACTTCCTGTAACAACGCA-3'
\end{tabular}

assess the change of intracellular lactate concentration in HT29 cells after CD147 silencing. Cells $\left(1 \times 10^{6}\right)$ were harvested by centrifugation and were then ruptured by hypotonic salt solution for $1 \mathrm{~h}$ at room temperature. The supernatant was retained after centrifuging. The optical density was read at $530 \mathrm{~nm}$. This experiment was repeated at least three times.

Cell proliferation assay. Cell proliferation in vitro was analyzed with the formazan substrate, 2-(2-methoxy-4nitrophenyl)-3-(4-nitrophenyl)-5 (2, 4-disulfophenyl)-2H tetrazolium monosodium salt (WST-8). Cells were plated in 96-well plates in $100 \mu \mathrm{l}$ DMEM at a density of $1 \times 10^{4}$ cells per well. After $24,48,72,96,120 \mathrm{~h}$ of culture, respectively, the medium was removed and replaced with fresh $100 \mu \mathrm{l}$ medium, then $10 \mu \mathrm{l}$ of WST- 8 was added to each well and the plates were returned to standard tissue incubator conditions for an additional $1.5 \mathrm{~h}$. Colorimetric analysis was performed at $450 \mathrm{~nm}$ wavelength on a micro plate reader. Each analysis was done at least three times.

Invasion assay. Transwell plates (Corning Costar, Cambridge, MA, USA) were coated with basement membrane Matrigel (20 mg/ml, Becton-Dickinson, Franklin Lakes, NJ, USA) for $4 \mathrm{~h}$ at $37^{\circ} \mathrm{C}$. Serum-free DMEM containing $1 \times 10^{5}$ cells in $100 \mu \mathrm{l}$ was added into the upper chamber, the lower chamber received $500 \mu \mathrm{l}$ of $10 \%$ FBS-containing medium and was incubated at $37^{\circ} \mathrm{C}$ for $24 \mathrm{~h}$. After $18 \mathrm{~h}$, cells that migrated through the permeable membrane were fixed with $100 \%$ methanol for $10 \mathrm{~min}$. The membranes with cells were soaked in $0.1 \%$ crystal violet for $10 \mathrm{~min}$ and then washed with distilled water. The number of cells which attached to the lower surface of the polycarbonate filter was counted at $\mathrm{x} 400$ magnification under a light microscope. Results were expressed as mean of triplicate experiments.

Drug sensitivity assay. To assess the antitumor drug chemosensitivity, cells were seeded in triplicates on 96-well plates at $1 \times 10^{4}$ cells/well and incubated for $24 \mathrm{~h}$. The medium was then removed and added with fresh medium containing cisplatin, paclitaxel, gemcitabine and oxaliplatin (Sigma) with varying concentrations: $0.1,1$ and $10 \mu \mathrm{M}$. After $48 \mathrm{~h}$, cells were treated with MST-8 as described earlier. Spectrometric absorbance at $450 \mathrm{~nm}$ was measured with a micro-plate reader. Each group was repeated at least three times.

In vivo tumor progression assay. Tumor xenografts were established by subcutaneous injection of $5 \times 10^{6}$ HT29, HT29/ shRNA-control, HT29/shRNA cells into the right flank of 4-6-week-old female nude mice, respectively. The experiments were approved by the Experimental Animal Center of University of Yangzhou, Yangzhou, China. The size of the transplanted tumors was measured every 5 days and the average tumor volume was measured: volume $=1 / 2 \times$ (length $\mathrm{x}$ width ${ }^{2}$ ). All animals were euthanized after 30 days postinoculation. Harvested tissues were fixed in $10 \%$ buffered formalin, embedded in paraffin, sectioned at $4 \mu \mathrm{m}$, and stained with H\&E. Immunohistochemistry analysis used goat anti-mouse CD147 polyclonal antibody (1:50 dilution, Santa Cruz Biotechnology) to detect CD147 protein expression. Animal experiments were performed in accordance with institutional guidelines for animal care by Nanjing Medical University.

Statistical analysis. Statistical analysis was performed by the SPSS software. Each assay was conducted at least three times. All experimental data were expressed as the mean $\pm \mathrm{SD}$ and assessed by Student's t-tests and one-way ANOVA at a significance level of $\mathrm{p}<0.05$. 


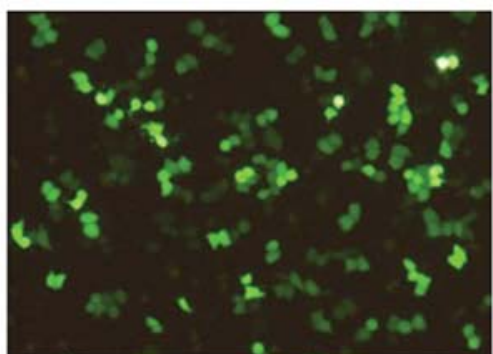

HT29/shRNA-control

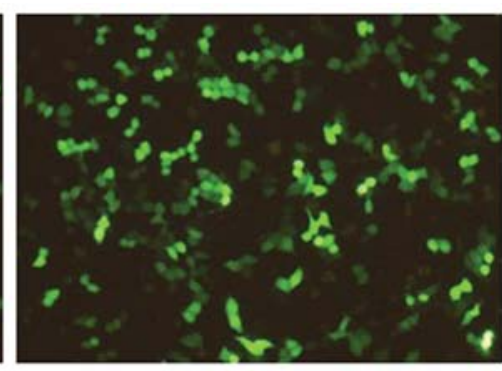

HT29/shRNA

Figure 1. Cell-specific expression of EGFP in HT29 cell lines transfected with pYr-mir30-shRNA vectors. The expression of EGFP in HT29/shRNAcontrol and HT29/shRNA cells is shown.

A
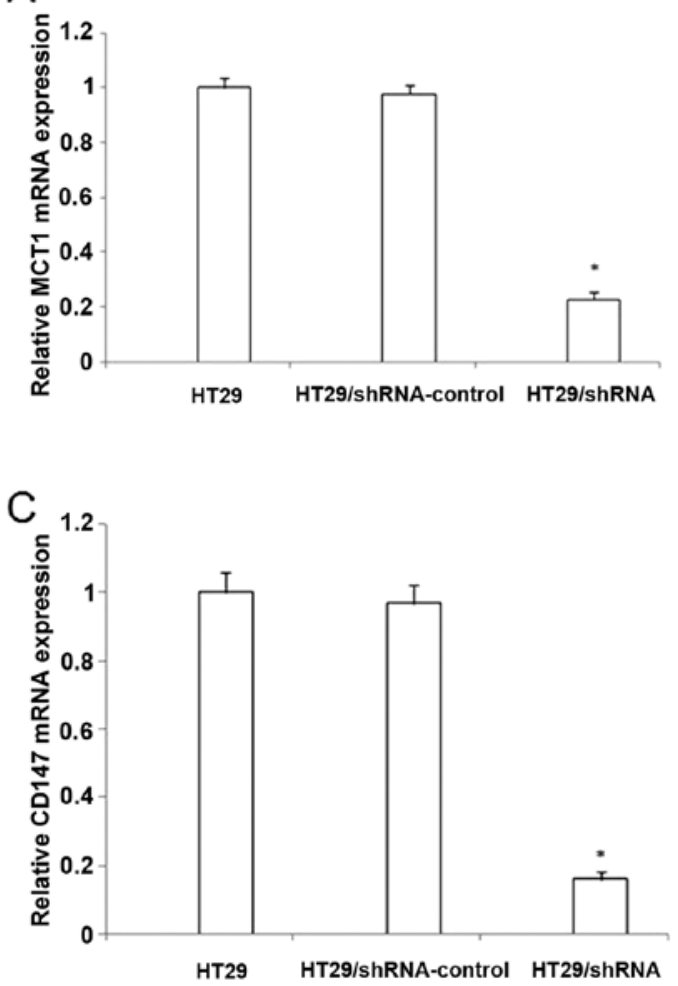

B

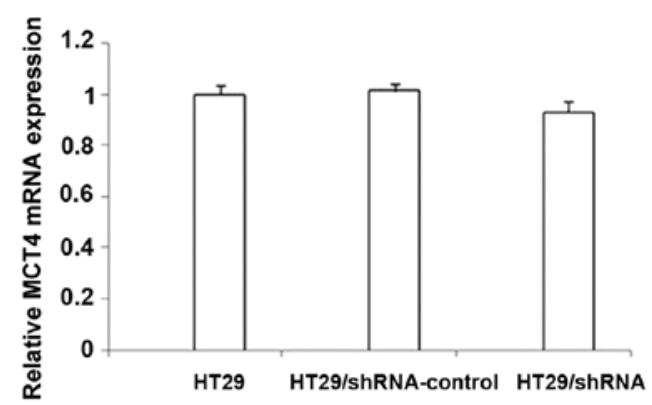

L1 L2 L3

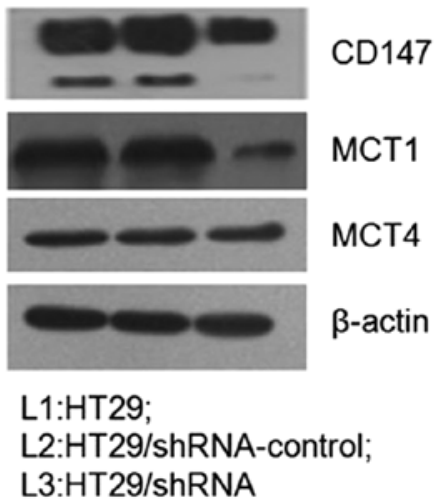

Figure 2. CD147 specific shRNA results in the reduction of CD147 mRNA and protein levels in HT29 cells. The expression levels of CD147, MCT1 and MCT4 in HT29 cells after CD147 silencing. Relative mRNA levels were analysed by quantitative RT-PCR. $\beta$-actin was used as normalization control. As shown in (A) and (C), the mRNA expression of CD147 and MCT1 were significantly downregulated (p<0.01) by HT29/shRNA compared with the control group in HT29 cells. (B) The mRNA expression of MCT4 had no significant change ( $>0.05$ ). The graphs are representative of three separate experiments. (D) The CD147, MCT1 and MCT4 protein expression levels by western blotting. The control group used $\beta$-actin. The results show that the protein expression levels of CD147 and MCT1 were significantly downregulated by HT29/shRNA in cells ( $<<0.01$ ). There was no significant change of MCT4 protein expression ( $>0.05$ ).

\section{Results}

Selection of pYr-mir30-shRNA stable expression transfectants. In our study, we established two vectors, the pYr-mir30-shRNA-control and the pYr-mir30-shRNA. After $48 \mathrm{~h}$ of transfect, the transfect cells were treated with $600 \mu \mathrm{g} /$ ml G418 selection. After two weeks, the stable expression of HT29/shRNA and HT29/shRNA-control cells were obtained. The EGFP expression could be clearly observed as shown in Fig. 1.
The pYr-mir30-shRNA-mediated gene silencing inhibits CD147 expression in HT29 cells. To evaluate the expression of CD147 in HT29 cells, we used the real-time RT-PCR (RT-PCR) and western blotting. The gene of $\beta$-actin was selected as the internal gene. A significantly reduced CD147 mRNA level for HT29/shRNA was achieved compared with untreated HT29 cells, respectively (p<0.01) (Fig. 2). In addition, western blot analysis confirmed the downregulation of CD147 protein by the HT29/shRNA (Fig. 2). 

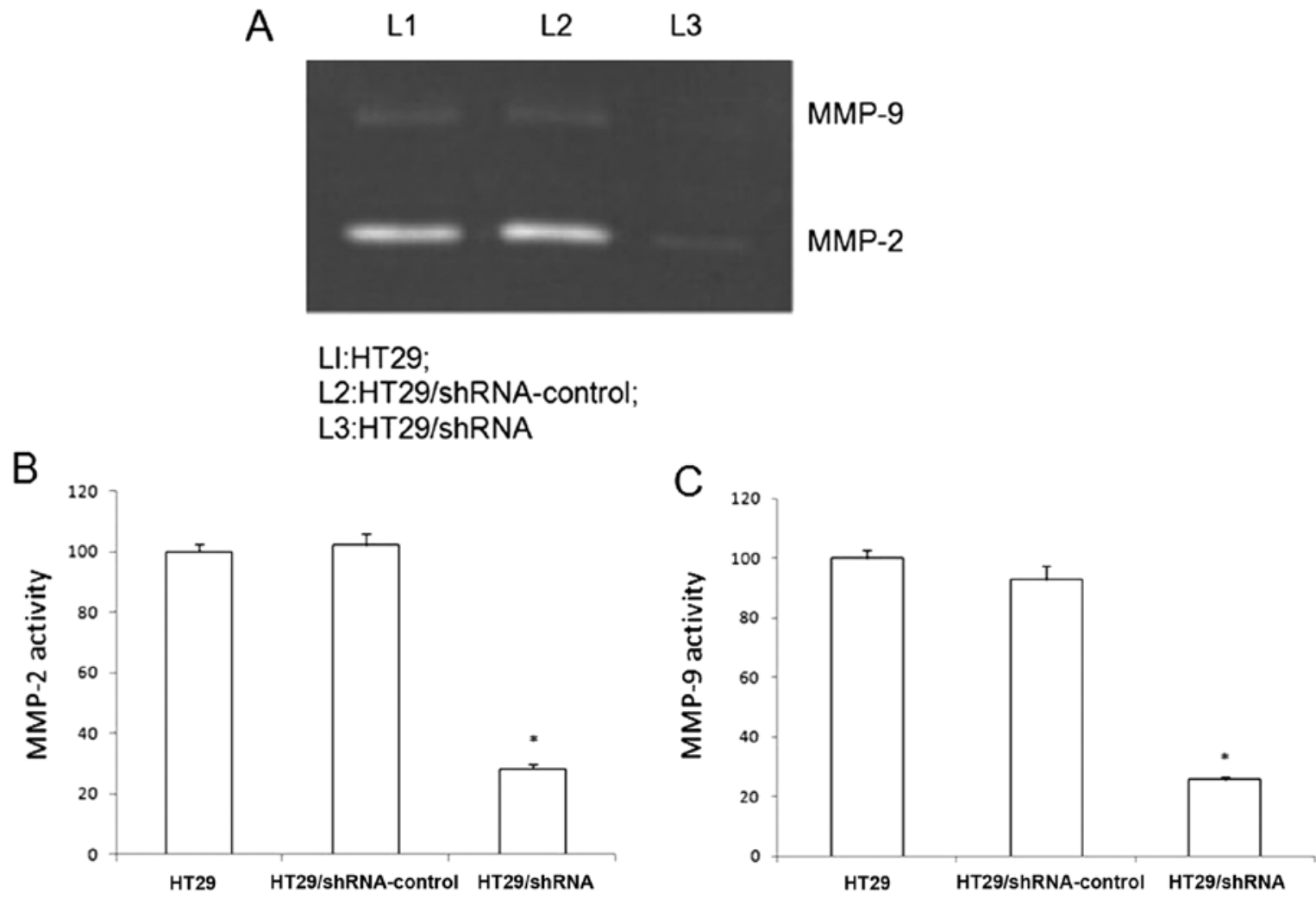

Figure 3. Gelatin zymography analysis of the activity of MMP-2 and MMP-9 in HT29 cells after CD147 silencing. Cells were incubated for $24 \mathrm{~h}$ and conditioned media were used for the measurement of MMP-2 and MMP-9 protein levels by gelatin zymography. (A) Images of the MMP-2 and MMP-9 bands representative of three independent experiments. (B) Quantitative analysis of the MMP-2 bands. "p<0.01 compared with HT29 and HT29/shRNA-control. (C) Quantitative analysis of the MMP-9 bands. " $\mathrm{p}<0.01$ compared with HT29.

The CD147 silencing inhibits MCT1 and MCT4 expression in HT29 cells. Many studies have confirmed the expression of MCT1 and MCT4 are closely associated with CD147 in various cancers. To detect whether the CD147 silencing could reduce the expression of MCT1 and MCT4. We performed real-time RT-PCR and western blotting. The real-time RT-PCR analysis, contrasted with the HT29/shRNA-control, the HT29/shRNA mRNA expression of MCT1 was downregulated $(\mathrm{p}<0.01)$, but the MCT4 mRNA expression did not significantly change in HT29 cells ( $>0.05$ ) (Fig. 2). In addition, western blot analysis demonstrated that the MCT1 protein was downregulation by HT29/shRNA ( $<<0.01)$, but the MCT4 protein did not significantly alter in HT29 cells ( $>00.05)$ (Fig. 2).

The CD147 silencing reduces the activity of MMP-2 and MMP-9 in HT29 cells. CD147 contributed to tumor invasion and metastasis by stimulating fibroblasts matrix metalloproteinase production. We used gelatin zymography to investigate the effect of CD147 silencing on HT29 cells reducing the activity of MMP-2 and MMP-9. As shown in Fig. 3, the secretion levels of MMP-2 and MMP-9 in the HT29/shRNA cells show significant difference in the HT29 cells $(\mathrm{p}<0.01)$. There was no significant difference between HT29 and HT29/ shRNA-control $(\mathrm{p}>0.05)$.

The CD147 silencing inhibits the function of lactate transporters in HT29 cells. We examined whether CD147

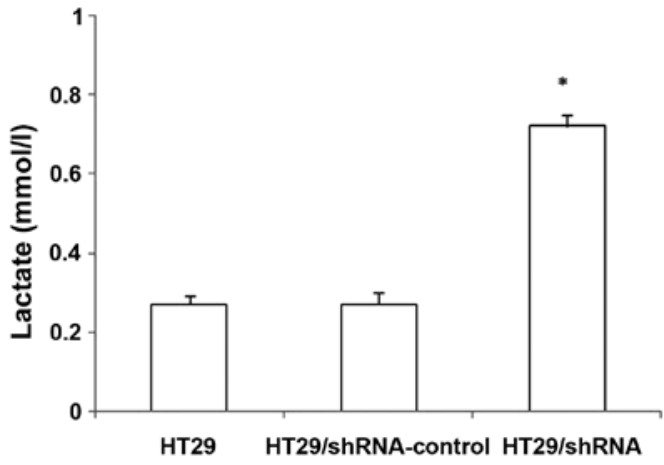

Figure 4. Intracellular lactate analysis in HT29 cells after CD147 silencing. The data show that the intracellular lactate concentration after transfection with HT29/shRNA was increased, ${ }^{*}$ p $<0.01$ compared with HT29.

silencing inhibited the function of lactate transporters used in the lactic acid assay. As shown in Fig. 4, the intracellular lactate concentration of the HT29 cells were increasing after CD147 silencing. There was no significant difference between HT29/shRNA-control and HT29 cells ( $>0.05$ ). These results confirmed that the downregulation of MCT1 expression by HT29/shRNA inhibits the function of these transporters in HT29 cells. Thus, the decrease of MCT1 expression is associated with an increase in intracellular lactate concentration.

The CD147 silencing reduces the proliferation of HT29 cells. In order to examine whether the CD147 silencing affects 


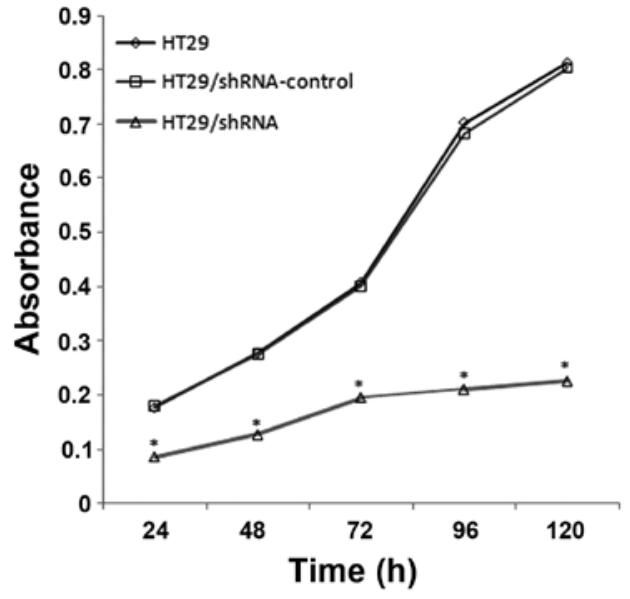

Figure 5. Proliferation of HT29 cells stably expressing shRNA. The proliferation of HT29 cells (HT29, HT29/shRNA-control, HT29/shRNA) was analyzed by WST- 8 assay. The spectrometrics absorbance at $450 \mathrm{~nm}$ was measured using a microplate reader. Each group contained three wells and the experiments were repeat three times (see Materials and methods). ${ }^{*} \mathrm{p}<0.01$ compared with HT29.

cell proliferation, we used the WST-8 assay to determine the proliferation of HT29, HT29/shRNA-control and HT29/ shRNA, respectively. The results (Fig. 5), compared with HT29, show the proliferation of HT29/shRNA was inhabited to $51.03(\mathrm{p}<0.01), 54.26(\mathrm{p}<0.01), 51.92(\mathrm{p}<0.01), 69.94(\mathrm{p}<0.01)$ and $72.24 \%(\mathrm{p}<0.01)$ at $24,48,72,96$ and $120 \mathrm{~h}$, respectively. There was no significant difference between HT29 and HT29/ shRNA-control $(\mathrm{p}>0.05)$.
CD147 silencing reduces the invasive ability of HT29 cells in vitro. To examine whether the downregulation of CD147 in HT29 cells affected its invasive ability, we performed a Matrigel Transwell analysis in vitro. The results showed that HT29 and HT29/shRNA-control cells had a similar ability to pass through the Matrigel coated filter (Fig. 6). The number of HT29/shRNA cells passing through the Matrigel was markedly lower than the numbers of HT29 and HT29/ shRNA-control cells $(\mathrm{p}<0.05)$.

The CD147 silencing increases the sensitivity to chemotherapeutic drugs in HT29 cells. CD147 was found to be overexpressed in multidrug resistance tumor cells and could confer resistance to some antitumor drugs. In order to test whether CD147 silencing affected its sensitivity to chemotherapeutic drugs in HT29 cells, we used WST-8 assay to investigate the sensitivity of HT29 cells to the antitumor drug. As shown in Fig. 7, the chemosensitivity of HT29 cells was significantly increased to the antitumor drug cisplatin $(\mathrm{p}<0.01)$, whereas to paclitaxel, gemcitabine or oxaliplatin, there was no significant difference between the HT29/shRNA and HT29/ shRNA-control after CD147 silencing $(\mathrm{p}>0.05)$.

CD147 silencing inhibited the tumor formation of HT29 cells in vivo. Using RNA interference can effectively reduce the proliferative ability of HT29 colorectal cancer cells in vitro, and we investigated its efficacy in vivo. From the date of vaccination, and every 5 days, we measured the tumor length and width and calculated their volume. As shown in Fig. 8A, the tumor growth was slower in the HT29/shRNA group than that in other groups $(p<0.01)$ and there was no significant

A
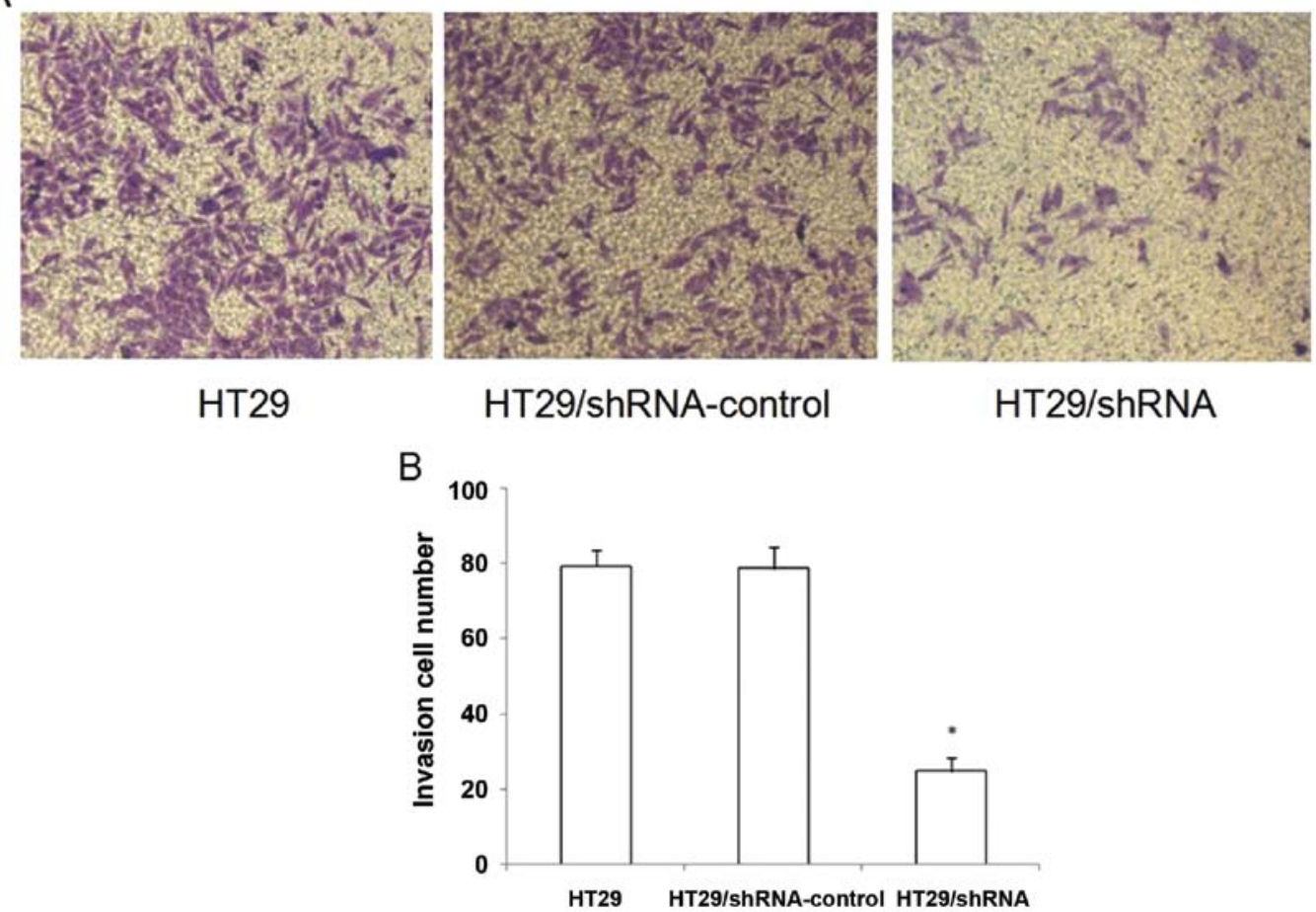

Figure 6. Invasive ability of HT29 cells on Matrigel after CD147 silencing. (A) Crystal violet staining results of lower surface filters show the cells invading the Matrigel (x400). (B) The number of cells that invaded through the chamber was evaluated in 3 fields for each experimental group and averaged. The invading cells of each experimental group was counted as the average of the sum of 10 fields of vision under a microscope. * $\mathrm{p}<0.05$ compared with HT29. 

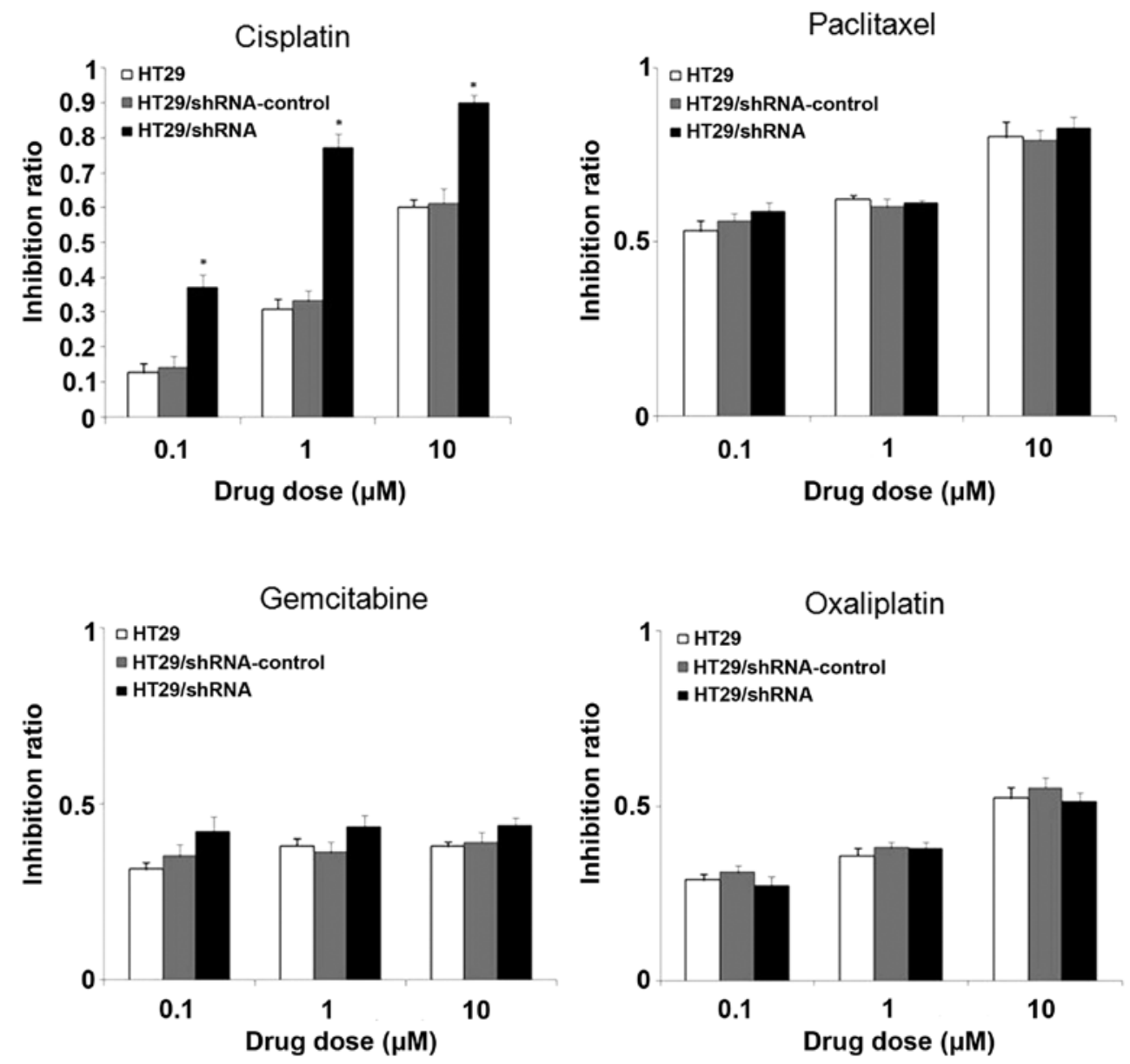

Figure 7. Multidrug chemosensitivity analysis in HT29 cells after CD147 silencing. Cells were treated with cisplatin with varying concentrations: $0.1,1$ and $10 \mu \mathrm{M}$ for $48 \mathrm{~h}$. Cell viability was determined by the WST-8 assay. CD147 silencing significantly increased the chemosensitivity of HT29 cells to cisplatin at $0.1,1$ and $10 \mu \mathrm{M}$ compared with the control groups $(\mathrm{p}<0.01)$. These experiments were repeated in three separate experiments. Cells were treated with paclitaxel, gemcitabine or oxaliplatin at varying concentrations: $0.1,1$ and $10 \mu \mathrm{M}$ for $48 \mathrm{~h}$ and determined by the WST-8 assay. There was no significant change of the chemosensitivity induced by CD147 silencing to paclitaxel, gemcitabine, and oxaliplatin in HT29 cells ( $>0.05$ ).

difference between HT29 and HT29/shRNA-control group ( $p>0.05)$. Hematoxylin and eosin (H\&E)-stained examination did not reveal obvious morphological changes among the tumors generated from the three groups, but there were larger areas of necrosis in the tumors formed by injecting with HT29/shRNA cells (Fig. 8B). Immunohistochemistry analysis showed that CD147 protein expression was high in HT29 and HT29/shRNA-control group mice, but was very low in tumors treated with HT29/shRNA (p<0.01) (Fig. 8B).

\section{Discussion}

CD147 is a multifunctional glycoprotein forming homooligomers in a cis-dependent manner in the plasma membrane (24). Based on the high CD147 expression reported in colorectal cancer and the association with colorectal development, we constructed an RNA interference vector, the pYr-mir30-shRNA. We used this vector to decline the CD147 expression in colorectal cancer cell line HT29 and further observed that the levels of CD147 mRNA and protein were significantly reduced, and the proliferation, invasion and metastasis of colorectal cancer HT29 cells were also reduced significantly.
CD147 was reported to be more highly expressed on the surface of most human carcinoma cells, and correlated with tumor progression and invasion by stimulating peritumoral fibroblasts to produce elevated levels of several MMPs (25). The present results showed that CD147 silencing resulted in a clear reduction of MMP-2 and MMP-9 expression in colorectal cancer cells, supporting the concept that CD147 was associated with increased expression of MMP-2 and MMP-9 (26). Data have suggested that CD147 stimulated the synthesis of specific MMPs to participate in tumor progression through peritumor fibroblasts (15). MMPs are believed to play important roles in disrupting the balance between growth and anti-growth signals in the tumor microenvironment $(27,28)$. Previous study reported that MMP-2 and MMP-9 were potential prognostic biomarkers of colorectal cancer (29). Development of a new generation of selective inhibitors of MMPs through the pharmacological targeting to colorectal cancer is a promising and challenging area for future research (30). In the present study, we also detected the HT29 cell invasion ability changes using transwell. The results showed that inhibition of CD147 expression reduced the ability of invasion in HT29 cells. The possible mechanism was that CD147 silencing inhibited the secretion of MMPs. In 

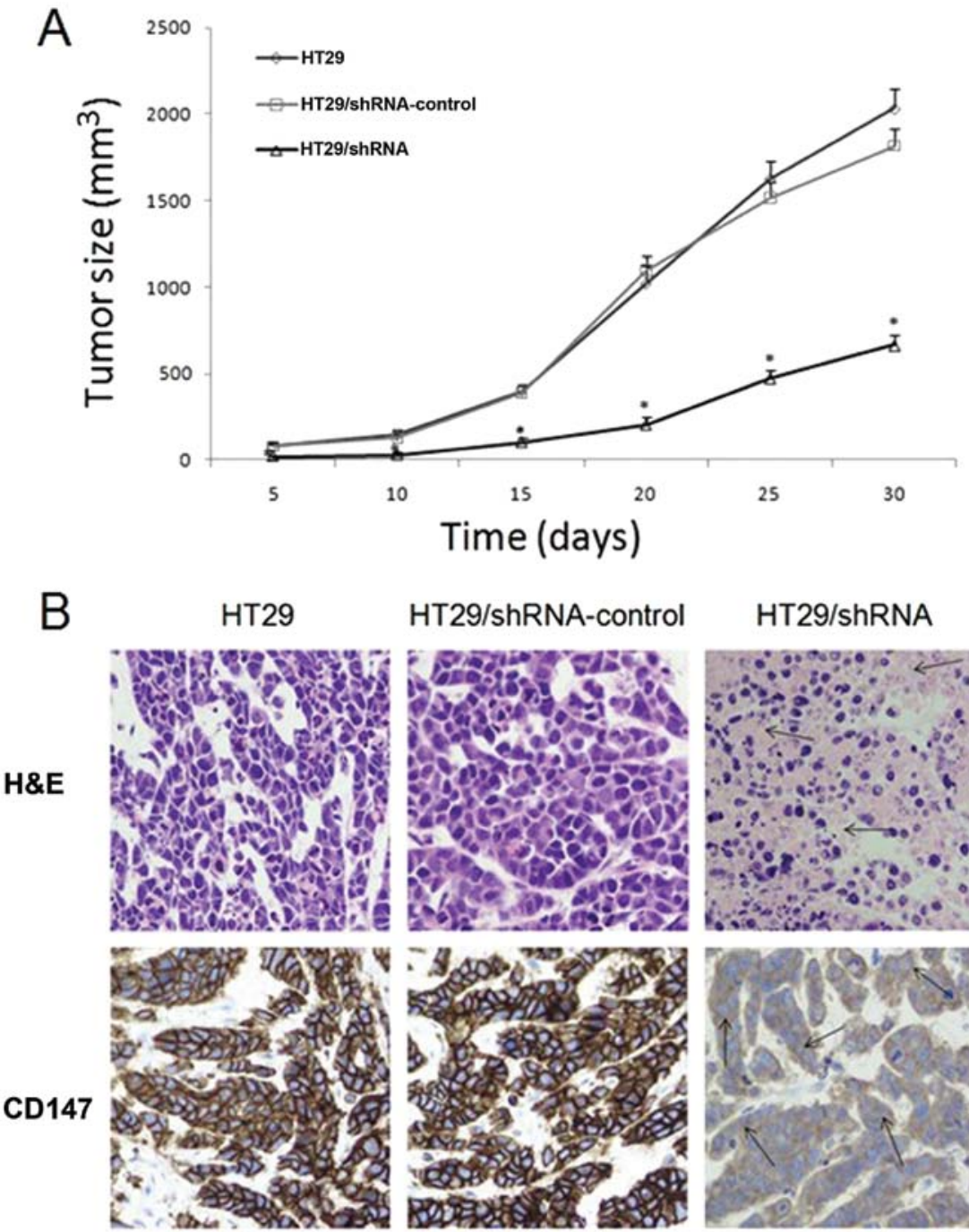

HT29/shRNA
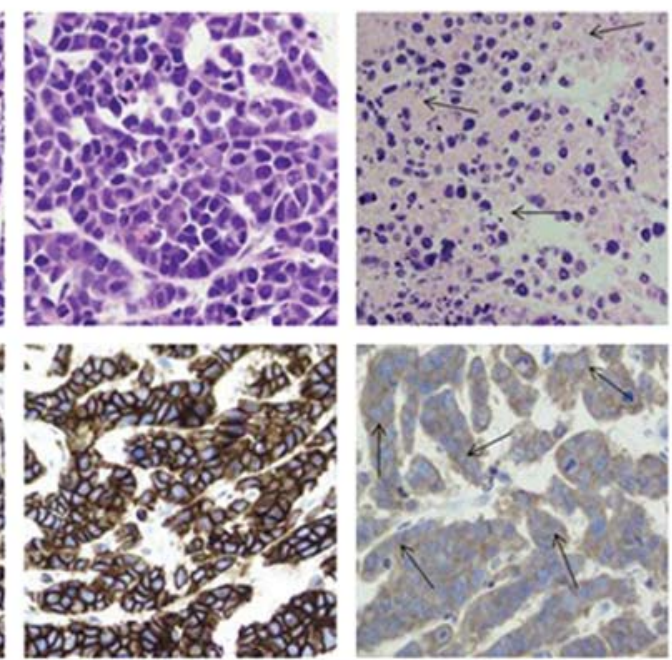

Figure 8. CD147 specific shRNA results on tumor formation in vivo and the expression of CD147 in tumor tissues. (A) The average size of the tumor in each group was measured every 5 days, by the formula: volume $=1 / 2 \times$ (length $\mathrm{x}$ width ${ }^{2}$ ). ${ }^{*} \mathrm{p}<0.01$ compared with HT29 group. (B) Immunohistochemistry staining in tumors to detect the CD147 protein expression (x400). The CD147 protein expression is shown in three different groups ( $\mathrm{x} 400$ ). " $\mathrm{p}<0.01$ compared with HT29 group. The data were obtained from three independent experiments. Histological analysis with H\&E staining was performed in implanted tumors (x400).

order to make the results of the experiment more convincing, we carried out animal experiments. The nude mouse experiments showed that inhibition of CD147 expression reduced colorectal cell tumorigenicity. Immunohistochemical staining suggested that CD147 protein expression was slightly detected in tumor derived from HT29/shRNA cells. These results proved that CD147 silencing could reduce the HT29 cell tumorigenicity, invasion and once again confirmed that the MMPs were associated with cell invasion.

CD147 was able to interact with certain lactate transporters (MCT1 and MCT4) and facilitate their expression on the cell surface. The present results showed that CD147 silencing resulted in a clear reduction of MCT1 expression, supporting the concept that $\mathrm{CD} 147$ was an ancillary protein required for the expression of these MCTs (21). Further evidence has demonstrated that the levels of CD147 in the plasma membrane were controlled by silencing or overexpressing of MCT4 (31). Our results showed that the CD147 silencing resulted in a significant reduction of MCT1, but the expression of MCT4 protein did not significantly change in colorectal cancer cells. CD147 inserted $\mathrm{H}^{+} /$lactate symporters, MCTs, in many organizations control the stability and function of plasma membrane, which played a determinant role in metabolic energy (32). In our study, CD147 silencing was able to increase the lactate concentration which might be due to the CD147 silencing leading to the MCT1 protein reduction, and the increase in lactate concentration might reduce the cell growth or other tumor-associated biological activities.

Multidrug resistance (MDR) occurred in tumor cells, tumor stem cells and tumor metastases, which is the main cause of failure in cancer therapy, and upregulated CD147 was observed in many MDR cancer cells (22). In colorectal cancer patients, MDR was also an important cause of treatment failure and mortality. In many different ways, the protein of CD147 could regulate the chemosensitivity of certain chemotherapeutic drugs $(33,34)$. The anticancer drugs cisplatin, paclitaxel, oxaliplatin and gemcitabine are widely used, and potent in head and neck cancer, and also often used in treatment of colorectal 
cancer. In the present study, the results revealed that CD147 silencing increased the chemosensitivity to cisplatin, but not to gemcitabine, oxaliplatin or paclitaxel in human colorectal cancer cell line HT29, suggesting that CD147 was an adjuvant chemotherapy target for colorectal cancer. Cisplatin was shown to be first efficacious compound in the treatment of colorectal cancer, and oxaliplatin consistently exerted antitumor activity in colorectal cancer $(35,36)$, the specific molecular mechanism of resistence to cisplatin are unclear, but several mechanisms of the resistance to cisplatin were proposed, such as reducing drug uptake, increasing drug inactivation, increasing DNA adduct repair and defecting apoptotic response, and we will investigate these mechanisms in the future.

In conclusion, CD147 silencing by RNAi inhibited the proliferation and invasion of cancer cells. The possible mechanism was that CD147 silencing inhibited the MCT1 protein expression, resulting in increased intracellular lactic acid and inhibition of cell proliferation. However, CD147 silencing suppressed the secretion of MMP proteins, thereby inhibited tumor cell invasion and metastasis. CD147 is a key regulator of the multidrug resistance of human colorectal cancer cell line HT29. The results of this study provide new ideas for potential strategies of gene target therapy in colorectal cancer.

\section{Acknowledgements}

This study was supported by National Nature Science Foundation of China (no. 81172141), Nanjing Science and Technology Committee project (no. 201108025), Nanjing Medical Technology Development Project (no. ZKX11025), Nanjing Health Young Talent Project, Jiangsu Provincial Key Medical Talents to S.K.W., Nanjing Medical Science and Technique Development Foundation to Y.Q.P. (no. QRX11255) and B.S.H. (no. QRX11254).

\section{References}

1. Ferlay J, Shin HR, Bray F, Forman D, Mathers C and Parkin DM Estimates of worldwide burden of cancer in 2008: GLOBOCAN 2008. Int J Cancer 127: 2893-2917, 2010.

2. Center MM, Jemal A, Smith RA and Ward E: Worldwide variations in colorectal cancer. CA Cancer J Clin 59: 366-378, 2009.

3. Stein U and Schlag PM: Clinical, biological, and molecular aspects of metastasis in colorectal cancer. Recent Results Cancer Res 176: 61-80, 2007.

4. Polette M, Gilles C, Marchand V, et al: Tumor collagenase stimulatory factor (TCSF) expression and localization in human lung and breast cancers. J Histochem Cytochem 45: 703-709, 1997.

5. Jiang JL, Zhou Q, Yu MK, Ho LS, Chen ZN and Chan HC: The involvement of $\mathrm{HAb} 18 \mathrm{G} / \mathrm{CD} 147$ in regulation of store-operated calcium entry and metastasis of human hepatoma cells. J Biol Chem 276: 46870-46877, 2001.

6. Riethdorf S, Reimers N, Assmann V, et al: High incidence of EMMPRIN expression in human tumors. Int J Cancer 119: 1800-1810, 2006.

7. Muramatsu T and Miyauchi T: Basigin (CD147): a multifunctional transmembrane protein involved in reproduction, neural function, inflammation and tumor invasion. Histol Histopathol 18: 981-987, 2003.

8. Rosenthal EL, Shreenivas S, Peters GE, Grizzle WE, Desmond R and Gladson CL: Expression of extracellular matrix metalloprotease inducer in laryngeal squamous cell carcinoma. Laryngoscope 113: 1406-1410, 2003.

9. Hanata K, Yamaguchi N, Yoshikawa K, et al: Soluble EMMPRIN (extra-cellular matrix metalloproteinase inducer) stimulates the migration of HEp-2 human laryngeal carcinoma cells, accompanied by increased MMP-2 production in fibroblasts. Arch Histol Cytol 70: 267-277, 2007.
10. Sameshima T, Nabeshima K, Toole BP, et al: Expression of emmprin (CD147), a cell surface inducer of matrix metalloproteinases, in normal human brain and gliomas. Int J Cancer 88: 21-27, 2000.

11. Stenzinger A, Wittschieber D, von Winterfeld M, et al: High extracellular matrix metalloproteinase inducer/CD147 expression is strongly and independently associated with poor prognosis in colorectal cancer. Hum Pathol 43: 1471-1481, 2012.

12. Zhu S, Chu D, Zhang Y, et al: EMMPRIN/CD147 expression is associated with disease-free survival of patients with colorectal cancer. Med Oncol 30: 369, 2013.

13. Pan Y, He B, Song G, et al: CD147 silencing via RNA interference reduces tumor cell invasion, metastasis and increases chemosensitivity in pancreatic cancer cells. Oncol Rep 27: 2003-2009, 2012.

14. Lynch CC and Matrisian LM: Matrix metalloproteinases in tumor-host cell communication. Differentiation 70: 561-573, 2002.

15. Caudroy S, Polette M, Tournier JM, et al: Expression of the extracellular matrix metalloproteinase inducer (EMMPRIN) and the matrix metalloproteinase- 2 in bronchopulmonary and breast lesions. J Histochem Cytochem 47: 1575-1580, 1999.

16. Caudroy S, Polette M, Nawrocki-Raby B, et al: EMMPRINmediated MMP regulation in tumor and endothelial cells. Clin Exp Metastasis 19: 697-702, 2002.

17. Biswas C, Zhang Y, DeCastro R, et al: The human tumor cellderived collagenase stimulatory factor (renamed EMMPRIN) is a member of the immunoglobulin superfamily. Cancer Res 55: 434-439, 1995.

18. Menashi S, Serova M, Ma L, Vignot S, Mourah S and Calvo F: Regulation of extracellular matrix metalloproteinase inducer and matrix metalloproteinase expression by amphiregulin in transformed human breast epithelial cells. Cancer Res 63: 7575-7580, 2003.

19. Guo H, Li R, Zucker S and Toole BP: EMMPRIN (CD147), an inducer of matrix metalloproteinase synthesis, also binds interstitial collagenase to the tumor cell surface. Cancer Res 60: 888-891, 2000.

20. Philp NJ, Ochrietor JD, Rudoy C, Muramatsu T and Linser PJ: Loss of MCT1, MCT3, and MCT4 expression in the retinal pigment epithelium and neural retina of the 5A11/basigin-null mouse. Invest Ophthalmol Vis Sci 44: 1305-1311, 2003.

21. Kirk P, Wilson MC, Heddle C, Brown MH, Barclay AN and Halestrap AP: CD147 is tightly associated with lactate transporters MCT1 and MCT4 and facilitates their cell surface expression. EMBO J 19: 3896-3904, 2000.

22. Yang JM, Xu Z, Wu H, Zhu H, Wu X and Hait WN: Overexpression of extracellular matrix metalloproteinase inducer in multidrug resistant cancer cells. Mol Cancer Res 1: 420-427, 2003.

23. Misra S, Ghatak S, Zoltan-Jones A and Toole BP: Regulation of multidrug resistance in cancer cells by hyaluronan. J Biol Chem 278: 25285-25288, 2003.

24. Yan L, Zucker S and Toole BP: Roles of the multifunctional glycoprotein, emmprin (basigin; CD147), in tumour progression. Thromb Haemost 93: 199-204, 2005.

25. Kanekura T, Chen $X$ and Kanzaki T: Basigin (CD147) is expressed on melanoma cells and induces tumor cell invasion by stimulating production of matrix metalloproteinases by fibroblasts. Int J Cancer 99: 520-528, 2002.

26. Zhu C, Pan Y, He B, et al: Inhibition of CD147 gene expression via RNA interference reduces tumor cell invasion, tumorigenicity and increases chemosensitivity to cisplatin in laryngeal carcinoma Hep2 cells. Oncol Rep 25: 425-432, 2011.

27. Lochter A and Bissell MJ: An odyssey from breast to bone: multi-step control of mammary metastases and osteolysis by matrix metalloproteinases. APMIS 107: 128-136, 1999.

28. Kessenbrock K, Plaks V and Werb Z: Matrix metalloproteinases: regulators of the tumor microenvironment. Cell 141: 52-67, 2010.

29. Roy R, Yang J and Moses MA: Matrix metalloproteinases as novel biomarkers and potential therapeutic targets in human cancer. J Clin Oncol 27: 5287-5297, 2009.

30. Herszenyi L, Hritz I, Lakatos G, Varga MZ and Tulassay Z: The behavior of matrix metalloproteinases and their inhibitors in colorectal cancer. Int J Mol Sci 13: 13240-13263, 2012.

31. Gallagher SM, Castorino JJ, Wang D and Philp NJ: Monocarboxylate transporter 4 regulates maturation and trafficking of CD147 to the plasma membrane in the metastatic breast cancer cell line MDA-MB-231. Cancer Res 67: 4182-4189, 2007. 
32. Le Floch R, Chiche J, Marchiq I, et al: CD147 subunit of lactate/ $\mathrm{H}^{+}$symporters MCT1 and hypoxia-inducible MCT4 is critical for energetics and growth of glycolytic tumors. Proc Natl Acad Sci USA 108: 16663-16668, 2011.

33. Zou W, Yang H, Hou X, Zhang W, Chen B and Xin X: Inhibition of CD147 gene expression via RNA interference reduces tumor cell invasion, tumorigenicity and increases chemosensitivity to paclitaxel in HO-8910pm cells. Cancer Lett 248: 211-218, 2007.

34. Jia L, Wang H, Qu S, Miao X and Zhang J: CD147 regulates vascular endothelial growth factor-A expression, tumorigenicity, and chemosensitivity to curcumin in hepatocellular carcinoma. IUBMB Life 60: 57-63, 2008.
35. Raymond E, Faivre S, Chaney S, Woynarowski J and Cvitkovic E: Cellular and molecular pharmacology of oxaliplatin. Mol Cancer Ther 1: 227-235, 2002.

36. Virag P, Perde-Schrepler M, Fischer-Fodor E, et al: Superior cytotoxicity and DNA cross-link induction by oxaliplatin versus cisplatin at lower cellular uptake in colorectal cancer cell lines. Anticancer Drugs 23: 1032-1038, 2012. 